\title{
Die Steuerpolitik als Mittel zur Durchsetzung der nationalsozialistischen Ziele
}

\subsection{Der Einfluss des politischen Wandels auf das deutsche Steuerrecht}

Die Machtübernahme der Nationalsozialisten erfolgte in mehreren Schritten und führte zur vollkommenen Aufhebung der Weimarer Reichsverfassung und ihrer demokratischen Kontrollen. ${ }^{88}$ Das „Ermächtigungsgesetz ${ }^{\text {“889 }}$, das der Reichstag mit der notwendigen Zweidrittelmehrheit gebilligt hatte, trat am 24. März 1933 in Kraft. Es erlaubte, den Reichstag auszuschalten, der Reichsregierung die volle Gesetzesmacht zu geben und die Gewaltenteilung abzuschaffen. Diese Kompetenzen fielen dem Reichskanzler zu, der sich für deren Durchführung Sonderbevollmächtigter bediente. ${ }^{890}$ Wenige Tage später, am 31. März 1933, folgten das „Gesetz zur Gleichschaltung der Länder mit dem Reich“ (1. Gleichschaltungsgesetz) und am 7. April 1933 das „Zweite Gesetz zur Gleichschaltung der Länder mit dem Reich“ (Reichsstatthaltergesetz). ${ }^{891}$ Damit waren die Länder aus der finanzpolitischen Willensbildung und aus der Finanzverwaltung ausgeschlossen, weil die Legislativund Exekutivfunktion bei den Reichsbehörden zentralisiert war.

Mit dem „Gesetz über den Neuaufbau des Reiches“vom 30. Januar $1934^{892}$ wurden die Volksvertretungen in den Ländern entfernt und die Hoheitsrechte auf das Reich übertragen. Damit wurde der im Weimarer Staat festgesetzte Finanzausgleich

888 Schauer, Ralf Erich: Die Steuergesetzgebung, S. 55.

889 Gesetz zur Behebung der Not von Volk und Reich („Ermächtigungsgesetz“), RGBl. I 1933, S. 141.

890 Benz, Wolfgang: Geschichte des Dritten Reiches, München 2000, S.25-27.

891 Overesch, Manfred/Saal, Friedrich Wilhelm: Chronik deutscher Zeitgeschichte, Bd. 2: Das Dritte Reich 1933-1939, Düsseldorf 1982, S. 38 und S. 42.

892 RGBl. I 1934, S. 75. 
zwischen Gesamtstaat und den Gliedstaaten faktisch gegenstandslos. ${ }^{893}$ Schließlich wurden den Ländern mit Gesetzen und Verordnungen im Laufe der Zeit sämtliche Steuerhoheiten entzogen, ${ }^{894}$ die Gemeinden hingegen erhielten mit Gesetz vom 10. Dezember 1937 die vormaligen Zuständigkeitsbereiche der Länder für die Grundsteuer, die Gewerbesteuer und die Bürgersteuer. ${ }^{895}$ Letztlich bestimmte allein der „Wille des Führers“ die Steuergesetzgebung, was zu einem verfassungsrechtlichen Zustand führte, der im neuzeitlichen Deutschland ohne Vorbild war. ${ }^{896}$

\subsection{Die Steuergesetzgebung im Nationalsozialismus}

Das Reichsfinanzministerium war als Teil der Regierung in die nationalsozialistische Politik integriert. An seiner Spitze standen von 1932-1945 Reichsfinanzminister Graf Schwerin von Krosigk und seit 1933 Fritz Reinhardt als Staatssekretär. Reinhardt besaß als „politischer Beamter“ als Einziger das Recht, Entscheidungen in Vertretung des Ministers zu treffen. Außerdem war er als „Beauftragter des Stellvertreters des Führers" für die Einhaltung der Grundsätze des Nationalsozialismus im gesamten Steuerrecht verantwortlich. ${ }^{897}$ In einer Sitzung der Akademie für Deutsches Recht in der Universität München verkündete Reinhardt am 26. Juni 1934 vor Parteigenossen seine vier Grundsätze nationalsozialistischer Steuerpolitik: ${ }^{898}$ Erstens: Oberstes Leitprinzip der Steuerpolitik ist die nationalsozialistische Weltanschauung. Zweitens: Die Steuerpolitik steht im Dienste der Verminderung

893 Voß, Reimer: Steuern im Dritten Reich. Vom Recht zum Unrecht unter der Herrschaft des Nationalsozialismus, München 1995, S. 18.

894 RGBl 1936, S. 315; RGBl 1942, S. 465; RGBl 1944, S. 282.

895 RGBl I 1937, S. 1351.

896 Voß, Reimer: Steuern im Dritten Reich, S.70

897 Pausch, Alfons: Persönlichkeiten der Steuerkultur. Steuergeschichte in Lebensbildern von Johannes von Miquel bis Armin Spitaler, Herne/Berlin 1992, S. 129.

898 RStBl 1934, S. 753 ff.; Reinhardt, Fritz: Die neuen Steuergesetze. Einführung in die neuen Steuergesetzesübersichten über die wesentlichen Änderungen gegenüber dem bisherigen Recht. Wortlaut der zehn neuen Gesetze, Berlin 1934; Ders.: Das Reinhardt-Programm. Die große nationalsozialistische Steuerreform. Vortrag von Fritz Reinhardt an der Universität München, Berlin 1934; Reinhard, Fritz: Leitsätze für die Gestaltung und Auslegung der Steuergesetze nach nationalsozialistischer Anschauung, in: Deutsche Steuer-Zeitung und Wirtschaftlicher Beobachter 24 (1935), S.625-633. Henning, Friedrich-Wilhelm: Die nationalsozialistische Steuerpolitik. Programm, Ziele und Wirklichkeit, in: Schremmer, Eckart (Hrsg.): Steuern, Abgaben und Dienste vom Mittelalter bis zur Gegenwart. Referate der 15. Arbeitstagung der Gesellschaft für 
der Arbeitslosigkeit. Drittens: Die Steuerpolitik hat familien- und volkspolitische Aufgaben zu erfüllen. Und viertens: Die Steuerpolitik soll den Wert der Persönlichkeit und der persönlichen Verantwortung in der Wirtschaft betonen.

Diese Grundsätze ließen sich jedoch nur durchführen, wenn jeder Steuerbeamte bedingungslos bereit war, sich der nationalsozialistischen Zielsetzung unterzuordnen. Eine Handhabe in personalpolitischer Hinsicht bot Reinhardt das „Gesetz zur Wiederherstellung des Berufsbeamtentums“ vom 7. April 1933. ${ }^{899}$ Das ermöglichte es den Finanzbehörden, sich von Mitarbeitern zu trennen, die im nationalsozialistischen Sinne als „politisch unzuverlässig“ galten. Das betraf in erster Linie Mitglieder linksgerichteter Parteien, die zwangspensioniert oder entlassen wurden, und Mitarbeiter jüdischer Geburt oder jüdischen Glaubens. Paragraf 3 dieses Gesetzes bestimmte, dass alle Beamten, „die nicht arischer Abstammung sind“, in den Ruhestand zu versetzen sind. Ausgenommen davon waren vorerst jene, die vor dem 1. August 1914 als Beamte tätig waren, im Ersten Weltkrieg auf deutscher Seite an der Front gedient hatten oder deren Väter oder Söhne in diesem Krieg gefallen waren. Dieses Gesetz hatte auch Konsequenzen für „Nichtarier“ unter den Steuerberatern, denn am 6. Mai 1933 wurde das „Gesetz über die Zulassung von Steuerberatern“ erlassen, das die Ausnahmen für Juden - die es für die Beamten gab - nicht enthielt, sodass allen jüdischen Steuerberatern das Recht auf Berufsausübung entzogen war. ${ }^{900}$ Zum Ausgleich wurden Mitglieder der NSDAP eingestellt und oftmals mit führenden Positionen betraut. Daneben baute Reinhardt ein neues Ausbildungssystem auf, das alle Steuerbeamten erfasste und diese auf die nationalsozialistische Steuerpolitik einschwor. ${ }^{901}$

\subsubsection{Die Steuerpolitik im Dienste der Verminderung der Arbeitslosigkeit}

Die ersten steuerlichen Maßnahmen wurden im Wesentlichen durch drei aufeinanderfolgende Gesetze bestimmt:

Sozial- und Wirtschaftsgeschichte vom 14. bis 17. April 1993 in Bamberg, Stuttgart 1994, S. 197-211, S. 202-207.

899 RGBl. I 1933, S. 175.

900 RGBl. I 1933, S. 257.

901 o. V.: Der neue Staatssekretär im Reichsfinanzministerium. Reinhardts Einführungsrede am 4. April 1933, in: Steuer-Warte 1933, S. 107. 
Das „Gesetz zur Verminderung der Arbeitslosigkeit“ vom 1. Juni $1933^{902}$ hatte ein Volumen von einer Milliarde Reichsmark und enthielt steuerliche Anreize zur Aktivierung der privaten Wirtschaftstätigkeit. Von besonderer Bedeutung war die Erweiterung des $₫ 16$ EStG, wonach Aufwendungen für die Anschaffung oder Herstellung von Wirtschaftsgütern des gewerblichen oder landwirtschaftlichen Anlagekapitals im Zeitpunkt der Anschaffung oder Herstellung - unter bestimmten Voraussetzungen - voll abgezogen werden konnten.

Am 15. Juli 1933 erließ das Reichskabinett das „Gesetz über Steuererleichterungen “. ${ }^{903}$ Es bestimmte, dass Instandsetzungsarbeiten und Ergänzungen an Betriebsgebäuden - allerdings nur bis 10 Prozent des Aufwands - steuermindernd geltend gemacht werden konnten. Ein Teil des Arbeitslohns konnte steuerfrei in Form von „Bedarfsdeckungsscheinen“ für Bekleidung und Hausrat ausgezahlt werden. Von besonderer volkswirtschaftlicher Bedeutung aber war, dass Unternehmen, die Erzeugnisse oder Produktionsmethoden entwickelten, von Steuerbehörden Steuernachlässe gewährt oder sie gar von der Steuer befreit werden konnten; die Bedingung war, dass dafür ein überragendes Bedürfnis der gesamten Gesellschaft vorlag.

Am 21. September 1933 wurde ein „Zweites Gesetz zur Verminderung der Arbeitslosigkeit “ ${ }^{\text {"904 }}$ erlassen. Dieses Gesetz enthielt Steuersenkungen und staatlichen Beihilfen in Höhe von 500 Millionen Reichsmark und sollte den privaten Wohnungsbau und die Landwirtschaft fördern.

Alle öffentlichen Maßnahmen zur Arbeitsbeschaffung sollten grundsätzlich der „Wiederwehrhaftmachung“ dienen, dies hatte Hitler bereits im Februar 1933 im Reichskabinett erklärt und dabei betont, dass dieser Gedanke immer und überall im Vordergrund stehen müsse. Arbeitsbeschaffung und Aufrüstung waren letztlich nur zwei Seiten der gleichen Medaille. Das erste Arbeitsbeschaffungsgesetz vom 1. Juni 1933 sah Steuervorteile insbesondere für die „Erhaltung und Erstarkung kriegswichtiger Betriebe“ vor. Beim zweiten Arbeitsbeschaffungsgesetz vom 21. September 1933 stand der Bau von Luftschutzräumen im Vordergrund. Der Ausbau der Autobahnen, des Schienennetzes und der Wasserwege erfolgte ebenfalls unter strategischen Gesichtspunkten. ${ }^{905}$

902 RGBl. I 1933, S. 323.

903 RGBl. I 1933, S. 491.

904 RGBl. I 1933, S. 651.

905 Ambrosius, Gerold: Von Kriegswirtschaft zu Kriegswirtschaft 1914-1945, in: North, Michael (Hrsg.): Deutsche Wirtschaftsgeschichte. Ein Jahrtausend im Überblick, München 2000, S. 287-355, S. 336. 


\subsubsection{Die Umgestaltung des aus der Weimarer Zeit übernommenen Steuersystems}

Nachdem 1933 die Reichsregierung auf steuerpolitischem Gebiet vor allem einzelne Maßnahmen getroffen hatte, die darauf abzielten, die Arbeitslosigkeit zu verringern und einigen Bevölkerungsgruppen, an denen den Nationalsozialisten politisch besonders gelegen war, gerecht zu werden, ging man 1934 dazu über, nationalsozialistische Grundsätze in die Steuergesetzgebung einzuführen. Dazu bot die Reichssteuerreform vom 16. Oktober 1934 eine besondere Gelegenheit. Die nationalsozialistischen Reformer behielten die Grundstruktur des Steuersystems bei, das der Reichsfinanzminister Matthias Erzberger 1919 eingeführt hatte, doch Staatssekretär Fritz Reinhardt führte mit Nachdruck eine Ideologisierung des Steuerrechts durch. ${ }^{906}$ Die Steuerreformgesetze vom 16. Oktober 1934 veränderten insgesamt zehn der damals geltenden Steuergesetze: das Einkommensteuer-, Körperschaftsteuer-, Umsatzsteuer-, Reichsbewertungs-, Erbschaftsteuer-, Vermögensteuer-, Kapitalverkehrsteuer- und Bürgersteuergesetz, das Gesetz über die Umwandlung von Kapitalgesellschaften sowie die Reichsabgabenordnung.

Steuerliches Grundgesetz des Reformwerks war das Steueranpassungsgesetz, das in seinen allgemeinen Vorschriften wesentliche Teile der Reichsabgabenordnung durch die "nationalsozialistische Weltanschauung“ ersetzte und damit auf sämtliche Steuergesetze übertrug. ${ }^{907}$

Im Steueranpassungsgesetz (StAnpG) vom 16. Oktober $1934^{908}$ heißt es in Paragraf 1:

(1) Die Steuergesetze sind nach nationalsozialistischer Weltanschauung auszulegen.

(2) Dabei sind die Volksanschauung, der Zweck und die wirtschaftliche Bedeutung der Steuergesetze und die Entwicklung der Verhältnisse zu berücksichtigen.

(3) Entsprechendes gilt für die Beurteilung von Tatbeständen.

Was unter „Beurteilung von Tatbeständen“ verstanden wurde, verdeutlichte Fritz Reinhardt in einer Rede, die er am 23. Oktober 1936 auf der Dritten Jahrestagung der Akademie für Deutsches Recht in München hielt:

Jede Gesetzesvorschrift kann im Einzelfall nur insoweit als maßgebend angesehen werden, als nicht besondere Tatumstände, die weder in der betreffenden noch in einer anderen Gesetzesvorschrift berücksichtigt sind, nach nationalsozialistischer

906 Sahm, Reiner: 5000 Jahre Steuern, S. 294.

907 Schauer, Ralf Erich: Die Steuergesetzgebung, S. 82.

908 RGBl. I 1934, S. 925. 
Weltanschauung eine andere Entscheidung bedingen. Es gibt kein Gesetz, dass nicht diesen inneren Vorbehalt enthält und infolgedessen nicht des Rechtswahrers als Gehilfen bedarf, um die nationalsozialistische Weltanschauung im Rechtsleben zu verwirklichen. Das für das Steuerrecht auszusprechen, ist der Zweck des $₫ 1$ Absatz 3 StAnpG. ${ }^{909}$

In Gesetzeskommentaren legte Reinhardt die steuerliche Generalvorschrift des $₫ 1$ StAnpG auch für Gesetze und Bestimmungen aus Zeiten vor dem 30. Januar 1933 aus: „Es sind demnach auch die Vorschriften des Handelsgesetzbuches nach nationalsozialistischer Weltanschauung auszulegen“. Es „,ist, kurz gesagt, alles richtig, was dem Volksganzen nützt, und alles falsch, was dem Volksganzen abträglich ist. " ${ }^{\text {"910 }}$

Damit war dem politischen Subjektivismus bei der Anwendung der Steuergesetze, wie auch aller anderen Gesetze, Tür und Tor für Diskriminierungen aus ideologischen und rassischen Gründen geöffnet. Was unter „nationalsozialistischer Weltanschauung “ zu verstehen war, war nirgendwo klar fixiert, sondern bestand aus einem Konglomerat von politischen Zielen, Verhaltensweisen und Aversionen. ${ }^{911}$ Auf der Grundlage dieser Generalklausel konnte „Unrecht sowohl durch strikte Beachtung des geltenden Rechts wie auch durch dessen Missachtung begangen werden". 912

Eine einschneidende Veränderung der alten Reichsabgabenordnung (RAO) in der Fassung vom 22. Mai $1931^{913}$ erfolgte durch den Paragrafen 21 StAnpG, mit dem Paragraf 12 der Reichabgabenordnung die folgende Fassung erhielt:

Der Reichsminister der Finanzen kann zur Durchführung und Ergänzung der vom Reich erlassenen Steuergesetze, insbesondere auch zur Überleitung der Gesetzgebung und der Behördenorganisation, Rechtsverordnungen und Verwaltungsvorschriften erlassen. Er kann insbesondere den Umfang der Befreiungen, Steuerermäßigungen und Steuervergütungen näher bestimmen.

Mit dieser Gesetzesveränderung entfiel Satz 2 des $\$ 12$ RAO in der Fassung vom 22. Mai 1931, nach dem der Reichsminister der Finanzen zum Erlass von steuerge-

909 Brönner, Herbert: Die Bilanz nach Handels- und Steuerrecht, Eberswalde/Berlin/Leipzig 1937, S. 89.

910 Reinhardt, Fritz: Beurteilung von Tatbeständen nach nationalsozialistischer Weltanschauung. Vortrag gehalten auf der Dritten Jahrestagung der Akademie für Deutsches Recht vom 23. Oktober 1936 in München, in: RStBl 1936, S. 1041-1056, S. 1044.

911 Sahm, Reiner: 5000 Jahre Steuern, S. 295.

912 Pausch, Alfons: Fritz Reinhardt als Mahnung. Ein Steuerfachmann im Dienste Hitlers, in: Der Steuerberater 11 (1987), S.349-360, S. 355.

913 RGBl I 1931, S. 161. 
setzlichen Bestimmungen grundsätzlich der Zustimmung des Reichrates bedurfte. Darüber hinaus konnte der Reichfinanzminister auch Vorschriften zur Ergänzung von Gesetzen erlassen. Die Ermessensentscheidungen des Reichsfinanzhofes wurden durch die Neufassung des $\$ 297$ RAO darauf begrenzt nachzuprüfen, ob sich Ermessensentscheidungen innerhalb der gesetzlich vorgegebenen Grenzen bewegten, während er bis dahin über Recht und Billigkeit von Ermessensentscheidungen zu bestimmen hatte. ${ }^{914}$

\subsubsection{Der Beitrag der Steuerpolitik zur Kriegsvorbereitung und Kriegsführung}

Die Reichssteuerreform vom 16. Oktober 1934 sollte nach Reinhardt nur den Beginn der nationalsozialistischen Steuerreform darstellen. ${ }^{915}$ Die Steuerpolitik der Jahre 1936 bis 1938 bestand in der Fortführung der Umgestaltung des Steuersystems nach nationalsozialistischen Grundsätzen. So wurde den Ländern ihr verbliebenes Besteuerungsrecht mit der Realsteuerreform, die durch das neue Grundsteuer- und Gewerbesteuergesetz zum 1. Dezember 1936 in Kraft trat, gänzlich entzogen. ${ }^{916}$ Personen, die nicht zur Erfüllung der zweijährigen aktiven Dienstpflicht einberufen wurden, wurden mit Gesetz vom 20. Juli $1937^{917}$ wehrsteuerpflichtig.

Kurze Zeit nach der Machtübernahme im Jahr 1933 wurden Maßnahmen ergriffen, um den Steuerzahler laufend zu kontrollieren. Dazu gehörte das am 12. Juni 1933 erlassene "Gesetz gegen Verrat der deutschen Volkswirtschaft" ${ }^{\text {“918, das }}$ eine Anzeigepflicht besonderer Art enthielt, denn es verpflichtete die Bürger, ihre Vermögensgegenstände im Ausland, die sie bisher dem Finanzamt verschwiegen hatten, anzugeben. Ebenso waren Devisen, die sie pflichtwidrig der Reichsbank nicht angeboten hatten, anzuzeigen. ${ }^{919}$

Um eine intensive Betriebsprüfung zu ermöglichen, wurden neue Prüfmethoden praktiziert. So verlangte die „Verordnung über die Führung eines Wareneingangsbuches“ vom 20. Juni 1935 in Artikel 1: „Gewerbliche Unternehmen (selbständige Handels- oder Gewerbetreibende einschließlich selbständige Handwerker jeder Art)

$914 \mathrm{Zu} \S 297 \mathrm{RAO}$ in Verbindung mit $\$ 21 \mathrm{Nr}$. 30 StAnpG vgl. Schauer, Ralf Erich: Die Steuergesetzgebung, S. 85 .

915 Vgl. RGBl. I 1934, S. 1236.

916 Schauer, Ralf Erich: Die Steuergesetzgebung, S. 127.

917 RGBl. I 1937, S. 821.

918 RGBl. I 1933, S. 360.

919 Sahm, Reiner: 5000 Jahre Steuern, S. 291. 
sind verpflichtet, für steuerliche Zwecke ein Wareneingangsbuch zu führen“ “920 Ein Jahr später, am 20. Juni 1936, wurde die „Verordnung über den Warenausgang ${ }^{\text {“921 }}$ erlassen. 1935 wurden Säumniszuschläge eingeführt, und 1936 wurde unter grober Missachtung des Steuergeheimnisses eine Liste säumiger Steuerzahler aufgestellt, die von jedermann eingesehen werden konnte. ${ }^{922}$

$\mathrm{Da}$ allein mit Steuermitteln die Aufwendungen für die Aufrüstung nicht zu bestreiten waren, musste ein immer größerer Prozentsatz der Ausgaben durch Kredite finanziert werden. Aus innen- und außenpolitischen Gründen wählte die Reichsregierung die Methode der „unsichtbaren“ Finanzierung. Dazu konzipierte der Reichswirtschaftsminister und Reichsbankpräsident Hjalmar Schacht den „Mefo-Wechsel“. Das waren fingierte Handelswechsel, mit denen die Höhe der Rüstungsausgaben verschleiert und das Reichsbankgesetz umgangen werden konnte. Zu diesem Zweck gründeten die Reichsbank und das Reichswehrministerium die Metallurgische Forschungsgesellschaft mbH (Mefo), deren Kapital die wichtigsten deutschen Rüstungsfirmen zeichneten. Es war eine Scheingründung mit der alleinigen Aufgabe, Wechsel von Rüstungslieferanten mit einer nach dem Reichsbankgesetz notwendigen zweiten "guten“ Unterschrift zu versehen. Insgesamt wurden in den Haushaltsjahren 1934/35 bis 1937/38 für 12 Mrd. Reichsmark Mefo-Wechsel ausgegeben, die ausschließlich der Aufrüstungsfinanzierung zugutekamen. ${ }^{923}$

Während bei der Änderung des Einkommensteuergesetzes von 1934 durch Gesetz vom 1. Februar $1938^{924}$ vor allem wirtschafts- und bevölkerungspolitische Korrekturen im Vordergrund standen, erfolgte mit Gesetz vom 17. Februar $1939^{925}$ eine diesmal kriegsbedingte Modifizierung. Das Ziel, die Einnahmen des Reiches zu steigern, wurde auch durch Körperschaftsteuererhöhungen mit den Gesetzen vom 27. August $1936^{926}$ sowie 25. Juli $1938^{927}$ verfolgt.

Eine weitere Maßnahme zur Einnahmesteigerung des Reiches war das „Gesetz über die Finanzierung nationalsozialistischer Aufgaben“. ${ }^{928}$ Es sah vor, dass zur Deckung des außergewöhnlich hohen Finanzbedarfs des Reiches grundsätzlich auf weitere Anleihen zu verzichten war, stattdessen sollten unverzinsliche Steu-

920 RGBl. I 1935, S. 752.

921 RGBl. I 1936, S. 507.

922 Runderlass des Reichsministers der Finanzen vom 04.12.1935, RGBl I 1935, S. 1489.

923 Petzina, Dietmar: Die deutsche Wirtschaft in der Zwischenkriegszeit, S. 119.

924 RGBl. I 1938, S. 99.

925 RGBl. I 1939, S. 283.

926 RGBl. I 1936, S. 701.

927 RGBl. I 1938, S. 952.

928 RGBl. I 1939, S. 561. 
ergutscheine ausgegeben werden. Die ersten dieser Steuergutscheine wurden am 2. Mai $1939^{929}$ ausgegeben; sie galten als Zahlungsmittel des Reiches zur späteren Verrechnung und ermöglichten die Finanzierung im Vorgriff auf spätere Steuereinnahmen. ${ }^{930}$

Die Lieferanten von Leistungen an Gebietskörperschaften oder an öffentliche Unternehmen erhielten 40 Prozent ihrer Forderungen in Steuergutscheinen, die sie bei den Vorlieferanten in Zahlung geben konnten. Reinhardt begründete diesen Weg wie folgt: „Die großen nationalpolitischen Aufgaben, die in der Gegenwart erfüllt werden müssen, [werden] auf Jahrzehnte verteilt, ohne daß dadurch eine Zinslast des Reiches verursacht wird. “931 Gleichzeitig mit den Steuergutscheinen wurde eine Mehreinkommensteuer durch Gesetz vom 30. März $1939^{932}$ eingeführt, eine Sondersteuer, um die angespannte Haushaltslage zu mildern.

Sowohl die Ausgabe von Steuerscheinen als auch die Mehreinkommensteuer wurden für die Zeit ab dem 1. April 1940 bzw. mit Verordnung vom 21. August 1940 mit Wirkung für das Jahr 1940 und die folgenden Kalenderjahre aufgehoben.

Mit Beginn des Krieges wurde durch Führererlass vom 30. August $1939^{933}$ der Ministerrat für die Reichsverteidigung ermächtigt, „Verordnungen mit Gesetzeskraft" auch für Steuergesetze zu erlassen. Auf diesem Wege ergingen das "Grundgesetz der Kriegswirtschaft" und die Kriegswirtschaftsordnung, die auch steuerliche Maßnahmen vorsahen. ${ }^{934}$

Diese Maßnahmen stellten sich wie folgt dar: ${ }^{935}$ Es wurden Kriegszuschläge auf bestimmte Steuerarten erhoben, und eine Dividendenabgabe wurde eingeführt. Fernerhin erfolgte eine gezielte Abschöpfung der Kaufkraft, indem Arbeitnehmern Steuerbefreiungen gewährt wurden, sofern diese für die Dauer des Krieges Sparkonten bei Kreditinstituten einrichteten, die erst zwölf Monate nach Kriegsende gekündigt werden konnten. Der Reichsfinanzminister war ermächtigt, von gewerb-

929 Steuergutscheine wurden bereits aufgrund der Notverordnung vom 4. 9.1932 für pünktliche Steuerzahlung und Mehreinstellung von Arbeitern, zur Belebung unternehmerischer Aktivität, ausgegeben.

930 Overesch, Manfred/Saal, Friedrich Wilhelm: Das Dritte Reich, S. 528.

931 Terhalle, Fritz: Geschichte der öffentlichen Finanzwirtschaft vom Beginn des 19. Jahrhunderts bis zum Schlusse des Zweiten Weltkriegs, in: Gerloff, Wilhelm/Neumark, Fritz (Hrsg.): Handbuch der Finanzwissenschaft, Bd. 1, 2. Aufl., Tübingen 1951, S. 273-326, S. 318 .

932 RGBl. I 1939, S. 561.

933 RGBl. I 1939, S. 1539.

934 Schauer, Ralf: Die Steuergesetzgebung, S. 121.

935 Ebd., S. 121-126. 
lichen Unternehmen, die eine außergewöhnliche Steigerung ihres Gewinns erzielt hatten, die Abführung eines bestimmten Betrages an das Finanzamt zu fordern.

\subsection{Die steuerliche Diskriminierung von Juden, "Zigeunern" und anderen „Fremdrassigen"}

Ein besonders großer Beitrag zur Finanzierung der Aufrüstung und der Kriegsführung wurde den Staatsbürgern jüdischer Abkunft, den „Zigeunern“ und anderen „Fremdrassigen“ abverlangt. Die Steuergesetzgebung war in der nationalen Rassenlehre begründet und trug in einer Fülle von ungerechten und unmenschlichen Gesetzen, Verordnungen und Erlassen letztlich dazu bei, den in Deutschland lebenden „Nichtariern“ jegliche Lebensgrundlage zu entziehen. Tempo und Umfang der Diskriminierungsmaßnahmen steigerten sich im Laufe der Zeit immer mehr. Nicht weniger als 312 steuerlich diskriminierende Gesetze und Verordnungen in der Zeit zwischen 1933 und $1945^{936}$ wurden erlassen; es soll hier nur auf die einschneidendsten steuerlichen Maßnahmen eingegangen werden.

Rechtfertigungsgrundlage dieses Vernichtungskampfes war das Parteiprogramm der NSDAP:

Staatsbürger kann nur sein, wer Volksgenosse ist. Volksgenosse kann nur sein, wer deutschen Blutes ist, ohne Rücksicht auf Konfession. Kein Jude kann daher Volksgenosse sein. [...] Wer nicht Staatsbürger ist, soll nur als Gast in Deutschland leben können und muß unter Fremdengesetzgebung stehen. ${ }^{937}$

Mit den Nürnberger Gesetzen vom 15. September 1935 schuf man mit dem Reichsbürgergesetz ${ }^{938}$ ein nur „Deutschblütigen“ zugängliches Reichsbürgerrecht und stempelte die Juden zu Staatsangehörigen zweiter Klasse. Mit einer in den Jahren 1937 und 1938 einsetzenden Welle neuer gesetzlicher Maßnahmen zielte man auf die Vernichtung der wirtschaftlichen Basis deutscher Juden ab. Einer der Endpunkte diskriminierender Maßnahmen bestand mit der elften Verordnung zum Reichsbürgergesetz ${ }^{939}$ darin, den deutschen Juden die Staatsangehörigkeit abzusprechen. ${ }^{940}$

936 Ebd., S. 142.

937 Voß, Reimer: Steuern im Dritten Reich, S. 31.

938 RGBl. I 1935, S. 1146.

939 RGBl. I 1941, S. 722.

940 Schauer, Ralf Erich: Die Steuergesetzgebung, S. 145-147. 


\subsubsection{Diskriminierungen auf dem Gebiet konventioneller Steuern}

Mit der Änderung des Einkommensteuergesetzes vom 1. Februar $1938^{941}$ schloss $\$ 32$ Nr. 3 EStG für jüdische Kinder die Kinderermäßigung aus. Regierungsrat Dr. Bacciocco aus dem Einkommensteuer-Referat beim Oberfinanzpräsidenten (OFP) Berlin-Brandenburg argumentierte ganz im Geiste des Nationalsozialismus: „Diese Maßnahme entspricht den rassepolitischen Grundsätzen des Nationalsozialismus und dem rassischen Empfinden des Deutschen Volks. ${ }^{{ }^{9}{ }^{42}}$

$\$ 21$ Abs. 3 der zweiten Einkommensteuerdurchführungsverordnung vom 17. März 1938 verhinderte eine Steuerermäßigung gemäß $\$ 33$ EStG, wenn außergewöhnliche Belastungen durch Kinder oder sonstige Angehörige jüdischen Glaubens entstanden waren..$^{943}$

Mit dem Einkommensteuer-Änderungsgesetz vom 17. Februar $1939^{944}$ wurde eine Sonderregelung für Juden bezüglich der verschiedenen Steuergruppen geschaffen. Jüdische Familien wurden vom Verheirateten-Tarif (Splittingtarif) ausgeschlossen.

Die Diskriminierung der Juden fand auch bei der Vermögensteuer ihren Niederschlag: Juden wurden ab dem Veranlagungszeitpunkt 1. Januar 1940 von sämtlichen Freibeträgen ausgeschlossen und waren bereits bei einem Vermögen von 5.000 Reichsmark - die reguläre Grenze betrug bei Verheirateten 20.000 Reichsmark und bei Ledigen 10.000 Reichsmark - zur Abgabe einer Vermögensteuererklärung verpflichtet. ${ }^{945}$

Jüdische Steuerpflichtige unterlagen mit der ersten Reichsmark der Umsatzsteuerpflicht, hingegen wurde anderen Reichsbürgern ein Freibetrag von 6.000 Reichsmark gewährt. ${ }^{946}$

941 RGBl. I 1938, S. 99.

942 Friedenberger, Martin: Fiskalische Ausplünderung. Die Berliner Steuer- und Finanzverwaltung und die jüdische Bevölkerung 1933-1945, Berlin 2008, S. 165.

943 Einkommensteuergesetz vom 17.03.1938, RGBl. I 1938, S. 503.

944 RGBl. I 1939, S. 283.

945 Verordnung zur Änderung des Vermögensteuergesetzes vom 31.10.1939, RGBl. I 1939, S. 2138.

946 RGBl. I 1938, S. 1935. 


\subsubsection{Auswanderung und Reichsfluchtsteuer}

Die Reichsfluchtsteuer, die am 8. Dezember 1931 von der Regierung Brüning eingeführt worden war, um die immense Kapitalflucht ins Ausland aufgrund der Auswirkungen der Weltwirtschaftskrise zu stoppen, wurde am 18. Mai $1934^{947}$ so abgefasst, dass sie sich vor allem gegen die zur Auswanderung entschlossenen jüdischen Bürger wendete.

Die Reichsfluchtsteuerverordnung von 1931 sah vor, dass deutsche Staatsangehörige mit einem Vermögen von über 200.000 Reichsmark oder alternativ einem Jahreseinkommen von mehr als 20.000 Reichsmark 25 Prozent ihres gesamten Vermögens als Reichsfluchtsteuer zu entrichten hatten, wenn sie ihren inländischen Wohnsitz aufgaben. Im Gesetz über Änderung der Vorschriften über die Reichsfluchtsteuer vom 18. Mai 1934 senkte die nationalsozialistische Reichsregierung den Grundfreibetrag auf ein Vermögen von 50.000 Reichsmark. Der Kreis der Steuerpflichtigen wurde damit beträchtlich ausgeweitet und betraf hauptsächlich Juden, die aus Furcht vor Gewalt ihr Heimatland verlassen wollten. Dieses Gesetz wurde während der nationalsozialistischen Herrschaft sechsmal verlängert und letztmals am 9. Dezember 1942 unbefristet fortgeschrieben. Nach diesen Gesetzen konnten Finanzämter sogar bei dem Verdacht auf eine geplante Ausreise eine Sicherheitsleistung in Höhe der geschätzten Reichsfluchtsteuer fordern. ${ }^{948}$

Die seit 1931 bestehende Devisenzwangsbewirtschaftung sorgte ab 1933 dafür, dass Emigranten einen immer kleineren Teil ihres Vermögens ins Ausland transferieren oder mitnehmen konnten. Da die Ausreisewilligen zu über 90 Prozent Juden waren, war ihnen auf diese Weise der Aufbau einer neuen Existenz im Ausland mit eigenen Mitteln verwehrt. Vom 1. Oktober 1934 an konnten nur noch Zahlungsmittel in Höhe von 10 Reichsmark ins Ausland mitgenommen werden, wobei von dieser Freigrenze nicht nur Zahlungsmittel, sondern auch Gold, Edelmetalle und Wertpapiere erfasst waren. ${ }^{949}$

Das Reichsfluchtsteueraufkommen betrug in den Jahren 1931 bis 1944 insgesamt 945 Millionen Reichsmark. ${ }^{950}$

947 RGBl. I 1934, S. 392.

948 Friedenberger, Martin: Fiskalische Ausplünderung, S. 84.

949 Ebd., S. 132.

950 Ebd., S. 71. 


\subsubsection{Judenvermögensabgabe}

Nachdem ein Gesetzentwurf im Jahr $1936^{951}$ - der eine umfassende Haftbarmachung des gesamten Judentums für Schäden, die einzelne Juden der deutschen Wirtschaft zufügten, vorsah - nicht verwirklicht wurde, ergab sich mit der Reichspogromnacht vom 9. November 1938 ein geeigneter Anlass, die wirtschaftliche Vernichtung der deutschen Juden herbeizuführen. Am 12. November $1938^{952}$ wurde die Verordnung über eine Sühneleistung der Juden deutscher Staatsangehörigkeit erlassen, die ihnen in ihrer Gesamtheit die Zahlung einer Kontribution von einer Milliarde Reichsmark auferlegte. Als offizielle Begründung wurde die Tötung des deutschen Diplomaten Ernst von Rath in Paris durch einen Juden namens Herschel Grynszpan angegeben, der Vergeltung für die von der deutschen Polizei durchgeführte Deportation seiner Eltern und Geschwister üben wollte. Im Reichsgesetzblatt vom 14. November 1938 lautete die Begründung für diese von Generalfeldmarschall Göring unterschriebene Verordnung: „Die feindliche Haltung des Judentums gegenüber dem deutschen Volk und Reich, die auch vor feigen Mordtaten nicht zurückschreckt, erfordert entschiedene Abwehr und harte Sühne. ${ }^{\text {"9953 }}$

Die Einziehung dieser Kontribution wurde den Finanzämtern übertragen. Die Zahlung von einer Milliarde Reichsmark sollte von jedem Juden anteilig als „Judenvermögensabgabe“ geleistet werden, der ein Mindestvermögen von 5.000 Reichsmark besaß mit einer Steuer in Höhe von 20 Prozent, die am 19. Oktober 1939 auf 25 Prozent auf das einzelne Vermögen erhöht wurde. ${ }^{954}$

Infolge des Novemberpogroms waren ca. 30.000 Juden in Konzentrationslager verschleppt worden, die aufgefordert wurden, von dort ihre Finanzamtsangelegenheiten zu regeln, was schlichtweg nicht möglich war. Viele wurden vor die Wahl gestellt, ihr Vermögen zur Verfügung zu stellen und sich damit zur Ausreise aus Deutschland zu verpflichten, oder in KZ-Haft zu verbleiben.

Die Finanzverwaltung nahm mit dieser Abgabe insgesamt 1.126.612.495 Reichsmark ein. Gedanken darüber, dass der über eine Milliarde Reichsmark hinausgehende Betrag wieder auszukehren sei, machte man sich im Reichsfinanzministerium nicht. ${ }^{955}$

951 Barkai, Avraham: Vom Boykott zur „Entjudung“. Der wirtschaftliche Existenzkampf der Juden im Dritten Reich 1933-1945, Frankfurt am Main 1988, S. 126.

952 RGBl. I 1938, S. 1579.

953 RGBl. I 1938, S. 1579.

954 RGBl. I 1939, S. 2059.

955 Friedenberger, Martin: Die Rolle der Finanzverwaltung bei der Vertreibung, Verfolgung und Vernichtung der deutschen Juden, in: Friedenberger, Martin u.a. (Hrsg.): 


\subsubsection{Entjudungsgewinnsteuer}

Mit der „Entjudungsgewinnsteuer “956 - auch „Arisierungs-“ oder „Ausgleichabgabe“ genannt - wurde die gesetzliche Grundlage für die „Arisierung" aller jüdischen Gewerbebetriebe geschaffen. Jüdische Inhaber von Betrieben konnten auf staatliche Anordnung hin gezwungen werden, ihren Betrieb weit unter dem Verkehrswert an Erwerber zu veräußern. Dabei partizipierte der Staat an zweierlei: Zum einen war es ganz im nationalsozialistischen Sinn, Juden erheblich zu benachteiligen, zum anderen hatte der Erwerber die Gewinnspanne aus der Differenz zwischen dem Verkehrswert und dem von staatlichen Abwicklern festgesetzten Veräußerungspreis zu versteuern. Das Steueraufkommen resultierte damit aus jüdischen Vermögensverlusten. ${ }^{957}$

\subsubsection{Sozialausgleichsabgabe}

Mit der zweiten Verordnung zur Durchführung der Verordnung über die Erhebung einer Sozialausgleichsabgabe vom 24. Dezember $1940^{958}$ hatten jüdische Steuerpflichtige einen fünzehnprozentigen Zuschlag zur Einkommen- und Lohnsteuer zu entrichten, eine Steuer, die seit dem 8. August 1940 für polnische Lohnarbeiter eingeführt worden war. Begründet wurde die Einführung der Sozialausgleichsabgabe mit einem „fehlenden Interesse aller Juden und Polen am Volksganzen“.959 Ab dem 1. April 1942 wurden „Zigeuner“ mit der Heranziehung zur Sozialausgleichabgabe den Juden und Polen gleichgestellt. ${ }^{960}$

Die Reichsfinanzverwaltung im Nationalsozialismus. Darstellung und Dokumente, Bremen 2002, S. 10-94, S. 19-21.

956 Erlass der Verordnung zur Ausschaltung der Juden aus dem deutschen Wirtschaftsleben vom 12.11.1938, RGBl. I 1938, S. 1580 sowie Verordnung über den Einsatz des jüdischen Vermögens am 03.12.1938, RGBl. I 1938, S. 1709.

957 Schauer, Ralf Erich: Die Steuergesetzgebung, S. $164 \mathrm{f}$.

958 RGBl. I 1940, S. 1666.

959 Oermann, Josef: Sozialausgleichsabgabe, Lohnausgleichsabgabe, Ostarbeiterabgabe, Berlin/ Wien 1942, S. 31.

960 RGBl. I 1942, S. 149; RStBl 1942, S. 453. 


\subsubsection{Die steuerliche Benachteiligung der übrigen „Fremdvölkischen"}

„Ostarbeiter“ - mit dem Begriff wurden sämtliche Arbeitskräfte aus den Gebieten der Sowjetunion in den Grenzen von 1937 versehen - wurden mit außerordentlich hohen Steuersätzen belastet. Begründet wurde dies einerseits damit, dass diese in ihrer Heimat wesentlich niedrigere Löhne beziehen würden, andererseits keine Beiträge zur Sozialversicherung bzw. zur Deutschen Arbeitsfront leisteten. Mit Wirkung zum 15. Juni 1942 wurde diese Steuer abgeschafft und durch eine „Ostarbeiterabgabe“ ersetzt, bei der der Unternehmer des beschäftigten „Ostarbeiters“ die Abgabe nach der Verordnung vom 30. Juni $1942^{961}$ abzuführen hatte. Erst ab dem 1. April 1944 wurden „Ostarbeiter“ den deutschen Lohnsteuerpflichtigen gleichgestellt..$^{962}$

\subsubsection{Kollektive Enteignung der deutschen Juden ${ }^{963}$}

Die Einziehung des Vermögens der deutschen Juden ordnete sich unmittelbar ein in das Ziel der NS-Politik eines judenfreien Deutschlands. Die totale Enteignung war durch einschlägige Regelungen verfügt, die nach den bereits angelaufenen Deportationen erlassen wurden. Das Reichfinanzministerium und die Steuer- und Zollbehörden waren Teil des bürokratisch organisierten nationalsozialistischen Unrechtsregimes. Mit der Vermögensverwertungsstelle beim Oberfinanzpräsidenten Berlin-Brandenburg wurde der Einzug des Vermögens rassisch und politisch Verfolgter durch das NS-Regime zwangsweise betrieben. An vielen Beispielen aus Finanzamtsakten zeigt Kurt Schilde diese „Bürokratie des Todes. “964

Am 29. Mai 1941 erging ein Führererlass über die Verwertung des eingezogenen Vermögens von Reichsfeinden. Mit dem Deportationserlass des Reichsfinanzministeriums vom 4. November 1941 wurde detailliert geregelt, wie mit dem Vermögen der aus Deutschland deportierten Juden verfahren werden sollte.

In der 11. Verordnung zum Reichsbürgergesetz vom 25. November 1941 wurde die Enteignung jüdischen Vermögens endgültig geregelt. Nach dieser Verordnung verloren emigrierte Juden und solche, die ihren "gewöhnlichen Aufenthalt“ im

961 RGBl. I 1942, S. 419.

962 RGBl. I 1945, S. 39.

963 Friedenberger, Martin: Die Rolle der Finanzverwaltung, S. 21-26.

964 Vgl. Schilde, Kurt: Bürokratie des Todes. Lebensgeschichten jüdischer Opfer des NS-Regimes im Spiegel von Finanzamtsakten, Berlin 2002. 
Ausland hatten oder dorthin „verlegten“, die deutsche Staatsangehörigkeit und mit ihr allen Besitz. Nach einer Anordnung des Reichsinnenministers wurden das Generalgouvernement und die übrigen besetzten Gebiete nach dem 25. November 1941 zum Ausland erklärt, sodass mit der Deportation automatisch der Verfall des Vermögens zugunsten des Fiskus eintrat. ${ }^{965}$

Vorab hatte sich die Gestapo bedient und versucht, sich einen möglichst großen Teil des Vermögens durch „Beiträge“, „Abgaben“ oder "freiwillige Spenden“ zu sichern. Was sich die Gestapo nicht vorab sicherte, fiel dem Fiskus zu. ${ }^{966}$

\subsection{Die Rolle des Reichsfinanzhofs}

In der Zeit der ersten steuerpolitischen Maßnahmen des Nationalsozialismus von 1933 bis 1935 stieg die Arbeitsbelastung der Steuergerichtsbarkeit als Folge von zahlreichen Notverordnungen und einer ansteigenden steuerlichen Belastung stark an. Reinhardt provozierte mit seiner Rede anlässlich der Einführung des neuen Präsidenten des Reichsfinanzhofes zudem einen Konflikt, indem er wörtlich ausführte: „Aus meinen Ausführungen über die Aufgaben des RFH ergibt sich zusammenfassend, daß im nationalsozialistischen Staat der RFH der Gehilfe des RdF [...] zu sein

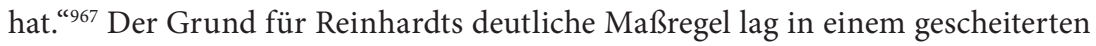
Versuch der Reichsregierung, der Judikative einen Weg vorzuschreiben, wie das Steuerrecht an den im Jahr 1934 verabschiedeten $₫ 1$ StAnpG angepasst werden konnte. Den Richtern bereitete die Anwendung des $\$ 1$ Abs. 3 StAnpG (Beurteilung von Tatbeständen nach nationalsozialistischer Weltanschauung) Probleme, denn danach war der Reichsfinanzhof verpflichtet, sich bei der Anwendung der vor 1933 erlassenen Steuergesetze zu vergewissern, ob diese mit den nationalsozialistischen Grundsätzen vereinbar waren. Der Reichsfinanzhof kam anfangs dieser Zumutung nicht widerspruchslos nach und riskierte Konflikte mit dem Ministerium; dann zog er sich bis 1938/39 auf die Linie zurück, im Grundsätzlichen standhaft zu bleiben, im Einzelnen jedoch den Wünschen des Ministeriums zu folgen. ${ }^{968}$

965 Ullmann, Hans-Peter: Der deutsche Steuerstaat, S. 165; bei den Deportationen schickte die Gestapo der Vermögensverwertungsstelle lediglich die Transportlisten mit sämtlichen Angaben jedes einzelnen zur Vernichtung bestimmten Juden mit dem Hinweis zu, dass dadurch das Vermögen der infrage stehenden Juden dem Reiche verfalle.

966 Ebd., S. 166.

967 RStBl. I 1935, S. 648.

968 Ullmann, Hans-Peter: Der deutsche Steuerstaat, S. 153. 
Reinhardt reagierte damit, dass er noch stärker als vorher Einfluss auf die personelle Besetzung der Ämter im Reichsfinanzhof nahm, was ab 1936 zur Folge hatte, dass sich Entscheidungen häuften, bei denen die Senate auf $₫ 1$ Abs. 3 StAnpG und den ,allgemeinen Beurteilungsgrundsatz" zurückgriffen. Der Konflikt endete mit einer fast vollständigen Beseitigung des Rechtsschutzes durch den Erlass des Führers vom 28. August $1939^{969}$, mit dem die Finanzgerichte - bis auf den Reichsfinanzhof - abgeschafft wurden. Während die bisher von den Finanzgerichten behandelten Fälle nun auf dem Verwaltungswege erledigt wurden, durfte der Reichsfinanzhof nur noch über grundsätzliche Fragen entscheiden. ${ }^{970}$

Durch weitere personelle Auswechslungen im Senat des Reichsfinanzhofes ab dem Jahr 1939 trat die antisemitische Grundhaltung ganz offen zutage. So entschied z. B. der RFH mit Urteil vom 20. November $1941^{171}$ über die Steuervergünstigung des ermäßigten Steuersatzes bei außerordentlichen Einkünften, die ein Jude in Anspruch nehmen wollte. Nach $\$ 34 \mathrm{EStG}$ unterlagen außerordentliche Einkünfte (z.B. Abfindungen, Gewinne aus Betriebsveräußerungen) einem besonderen Steuersatz, der grundsätzlich 10 Prozent betragen sollte. Auf Juden sollte dies keine Anwendung finden, wie der IV. Senat des Reichsfinanzhofes im Urteil entschied:

Es ist die Frage zu entscheiden, wie der vorliegende Tatbestand nach nationalsozialistischer Weltanschauung zu beurteilen ist ( $\$ 1$ Absatz 3 StAnpG). Es würde der gesunden deutschen Weltanschauung widersprechen, wenn einem Juden der ermäßigte Steuersatz [...] zugebilligt würde.

Der Reichfinanzhof entwickelte - trotz mancher Unterschiede zwischen den Senaten und einzelnen Richtern - „eine eigenartige Symbiose fiskalischer Engherzigkeit und nationalsozialistischer Weltanschauung “.972

969 RGBl. I 1939, S. 1535, Abschnitt IV, Abs. 4 und 5.

970 Schauer, Ralf Erich: Die Steuergesetzgebung, S. $204 \mathrm{f}$.

971 RFHE 51, S. 112.

972 Kumpf, Johann Heinrich: Der Reichsfinanzhof und seine Rechtsprechung in steuerlichen Angelegenheiten von Juden, in: Schönknecht, Ehrhardt (Hrsg.): Die Reichsfinanzverwaltung im Nationalsozialismus, Bremen 2002, S. 145; Uhlmann, Hans-Peter: Der deutsche Steuerstaat, S. 153. 


\subsection{Zusammenfassung und Fazit}

Mit dem „Gesetz zur Behebung der Not von Volk und Reich“ vom 23. März 1933 war der Weg geebnet, die Besteuerung des Reichs, der Länder und der Kommunen auf eine breite politisch-gesellschaftlich-wirtschaftliche Basis zu stellen. Die neue Steuerpolitik stand unter dem obersten Leitprinzip der "nationalsozialistischen Weltanschauung". Vom Standpunkt einer organischen Gesellschafts- und Staatsbetrachtung konnte das Postulat der Steuergerechtigkeit unbeachtet bleiben; denn wer grundsätzlich das Wohl des Ganzen voranstellte, sah die Individuen nur als abhängige Glieder, deren individuelle Interessen hinten anzustehen hatten.

\section{Zum Grundsatz der Allgemeinheit der Besteuerung}

Der Grundsatz der Allgemeinheit der Besteuerung wurde durch Hitler selbst durchbrochen. Mit einer geheimen Anordnung vom 19. Dezember 1934 sorgte er dafür, dass er selbst von der Steuerpflicht befreit wurde. Als Grund für diese Steuerfreiheit wurde die staatsrechtliche Stellung Hitlers als Führer des deutschen Volkes angeführt, die eine Steuerpflicht nicht begründen könne. ${ }^{973}$ Trotz der hohen Honorare aus schriftstellerischer Arbeit durch das zweibändige Werk „Mein Kampf", aus Einnahmen aus dem Recht am eigenen Bild und seinem Staatsgehalt als Reichskanzler und ab 1934 auch als Nachfolger des Reichspräsidenten wurden alle bis 1933 existierenden Steuerbescheide Hitlers für nichtig erklärt.974

Die steuerliche Diskriminierung der in Deutschland lebenden Juden war nicht nur ein Bruch des Allgemeinheits- und Gleichmäßigkeitssatzes, sondern war auch ein eklatanter Verstoß gegen die Freiheitsrechte. ${ }^{975}$ Mit den Nürnberger Gesetzen wurden Juden zu Staatsbürgern zweiter Klasse gestempelt, und mit der elften Verordnung zum Reichsbürgergesetz wurde den deutschen Juden die Staatsangehörigkeit abgesprochen.

\section{Zum Grundsatz der Gleichmäßigkeit der Besteuerung}

Da die nationalsozialistische Weltanschauung offiziell zu einem entscheidenden Kriterium für die Auslegung von Steuergesetzen, der Beurteilung von Tatbestän-

973 Pausch, Alfons: Wir prüfen Hitlers Steuerakten. Ein praktischer Fall aus der Steuergeschichte, in: Steuer und Studium, Zeitschrift für die Aus- und Fortbildung im Steuerrecht 9 (1988), S. 226-231.

974 Pausch, Alfons: Wie sich Hitler der Steuerpflicht entzog, in: Deutsches Steuerrecht. Zeitschrift für Praxis und Wissenschaft des gesamten Steuerrechts 21 (1983), S. 499-502.

975 Davidsohn, Lars: Verfassungsrechtliche Würdigung, S. 323. 
den und dem Treffen von Ermessensentscheidungen erklärt worden war, wurden die Gesetzmäßigkeit und Gesetzesbestimmtheit - und damit der Grundsatz der Gleichmäßigkeit in der Besteuerung - untergraben. ${ }^{976}$

Das Steueranpassungsgesetz war gleichbedeutend mit einer Generalvollmacht für die Finanzbehörden und bildete die Grundlage für alle folgenden Maßnahmen gegenüber Juden und anderen völkischen Minderheiten auf steuerlichem Gebiet. ${ }^{977}$ Eine nahezu endlose Kette steuerlich diskriminierender Gesetze und Verordnungen in den Jahren zwischen 1933 und 1939 wurde erlassen, die den Grundsatz der Gleichmäßigkeit der Besteuerung mit Füßen trat. Allein das Reichsfluchtsteueraufkommen betrug schon 1934 das mehr als 38-fache des Aufkommens im letzten Jahr der Weimarer Republik. ${ }^{978}$ Mit der Judenvermögensabgabe wurde der Gleichheitsgrundsatz in extremer Weise in sein Gegenteil verkehrt; die „Judenmilliarde“ trug wesentlich dazu bei, den im Herbst bevorstehenden Staatsbankrott zu verhindern.

Die nationalsozialistische Rassenlehre fand auch in der Rechtsprechung Niederschlag. Die massive Beeinflussung der Gerichtsbarkeit führte zu einer erheblichen Beeinträchtigung einer Besteuerung, die dem Grundsatz der Gleichmäßigkeit entsprechen sollte.

\section{Zum Prinzip der Besteuerung nach der Leistungsfähigkeit}

Die nationalsozialistische Steuerpolitik diente zwei Zielen: Zum einen sollten mehr Geldmittel zur Finanzierung der Aufrüstung und später des Krieges beschafft werden, ${ }^{979}$ zum anderen sollte dies möglichst zulasten solcher Organisationen und Personengruppen geschehen, die nach der nationalsozialistischen Rassenlehre Menschen zweiter Klasse waren. ${ }^{980}$

Für die Nationalsozialisten waren „Nicht-Arier“ Menschen ohne Recht, auf die das Prinzip der Besteuerung nach der Leistungsfähigkeit nicht anwendbar war.

976 Tipke, Klaus: Die Steuerrechtsordnung, S. 137, Anm. 74; Voß, Reimer: Steuern im Dritten Reich, S.238.

977 Longerich, Peter: Politik der Vernichtung. Eine Gesamtdarstellung der nationalsozialistischen Judenverfolgung, München/Zürich 1978, S. 125.

978 Jagdfeld, August: Steuerflucht und Steuerfluchtbekämpfung von Brüning bis Brandt, in: Steuer und Wirtschaft 49 (1972), S. 258-263, S. 258.

979 Von den 101,5 Milliarden Reichsmark, die das Deutsche Reich nach Schätzungen des Reichsfinanzhofes zwischen 1933 und 1939 ausgegeben hatte, fielen $60 \%$ auf Rüstungsausgaben; vgl. Petzina, Dietmar: Die deutsche Wirtschaft in der Zwischenkriegszeit, S. 118 .

980 Schauer, Ralf Erich: Die Steuergesetzgebung, S. 249 f. 
Zahlreiche Sondervorschriften auf dem Gebiet konventioneller Steuern stellten entsprechend eklatante Verstöße gegen den Leistungsfähigkeitsgrundsatz dar. ${ }^{981}$

\section{Zum Redistributionspostulat oder der Grundsatz der steuerlichen Umverteilung von Einkommen und Vermögen}

Die von Reinhardt 1934/1935 angestrebte Entlastung und Umverteilung ${ }^{982}$ war ausschließlich Ausfluss der nationalsozialistischen Ideologie. Die Steuerpolitik hatte einerseits familien- und bevölkerungspolitische Aufgaben zu erfüllen, was sich u. a. in höheren Freibeträgen bei Verheirateten und für Kinder sowohl bei der Einkommensteuer, als auch bei der Erbschaft- und Vermögensteuer niederschlug. Ledige wurden hingegen zusätzlich belastet. Die „nationalsozialistische Weltanschauung" öffnete andererseits Tür und Tor für die steuerliche Diskriminierung von Juden, „Zigeunern“ und anderen „Fremdrassigen“, die durch Sondersteuern, Sonderabgaben und Vermögenskonfiskationen fiskalisch gewaltsam belastet wurden.

Dieses Buch wird unter der Creative Commons Namensnennung 4.0 International Lizenz (http://creativecommons.org/licenses/by/4.0/deed.de) veröffentlicht, welche die Nutzung, Vervielfältigung, Bearbeitung, Verbreitung und Wiedergabe in jeglichem Medium und Format erlaubt, sofern Sie den/die ursprünglichen Autor(en) und die Quelle ordnungsgemäß nennen, einen Link zur Creative Commons Lizenz beifügen und angeben, ob Änderungen vorgenommen wurden.

Die in diesem Buch enthaltenen Bilder und sonstiges Drittmaterial unterliegen ebenfalls der genannten Creative Commons Lizenz, sofern sich aus der Abbildungslegende nichts anderes ergibt. Sofern das betreffende Material nicht unter der genannten Creative Commons Lizenz steht und die betreffende Handlung nicht nach gesetzlichen Vorschriften erlaubt ist, ist für die oben aufgeführten Weiterverwendungen des Materials die Einwilligung des jeweiligen Rechteinhabers einzuholen.

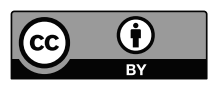

981 Voß, Reimer: Steuern im Dritten Reich, S. 237 f. und S. 244.

982 Zur Definition der steuerlichen Umverteilung von Einkommen und Vermögen, wie sie hier zugrunde gelegt wird, vgl. Neumark, Fritz: Grundsätze, S. 195, vgl. Kapital 2.3 letzter Absatz. 